Acta Crystallographica Section D

\section{Biological Crystallography}

ISSN 0907-4449

\section{Magdalena Schacherl, ${ }^{\mathrm{a}, \mathrm{b}}$ Sandro Waltersperger $^{b, c}$ and Ulrich Baumann $^{\mathrm{a}, \mathrm{b}_{*}}$}

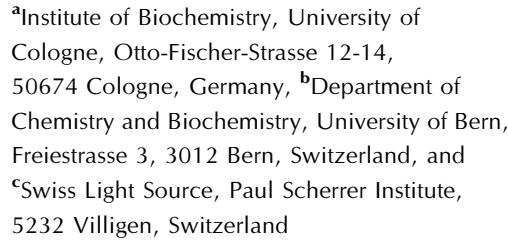

Correspondence e-mail: ubaumann@uni-koeln.de
(C) 2013 International Union of Crystallography Printed in Singapore - all rights reserved

\title{
Structural characterization of the ribonuclease H-like type ASKHA superfamily kinase MK0840 from Methanopyrus kandleri
}

Murein recycling is a process in which microorganisms recover peptidoglycan-degradation products in order to utilize them in cell wall biosynthesis or basic metabolic pathways. Methanogens such as Methanopyrus kandleri contain pseudomurein, which differs from bacterial murein in its composition and branching. Here, four crystal structures of the putative sugar kinase MK0840 from M. kandleri in apo and nucleotide-bound states are reported. MK0840 shows high similarity to bacterial anhydro- $N$-acetylmuramic acid kinase, which is involved in murein recycling. The structure shares a common fold with panthothenate kinase and the 2-hydroxyglutaryl-CoA dehydratase component $\mathrm{A}$, both of which are members of the ASKHA (acetate and sugar kinases/Hsc70/actin) superfamily of phosphotransferases. Local conformational changes in the nucleotide-binding site between the apo and holo forms are observed upon nucleotide binding. Further insight is given into domain movements and putative active-site residues are identified.

\section{Introduction}

Peptidoglycan, or murein, is responsible for the shape and the stability of the eubacterial cell and exhibits various biological activities (Stewart-Tull, 1980; Oken et al., 1981; Atrih \& Foster, 1999). It is targeted by small molecules such as $\beta$-lactam antibiotics, which block the enzymes responsible for cell-wall synthesis, for example transpeptidases and transglycosylases. Murein consists of two amino sugars, $N$-acetylglucosamine (GlcNAc) and $N$-acetylmuramic acid (MurNAc), connected by a $\beta$ - $(1,4)$-glycosidic bond. In most Gram-positive bacteria MurNAc is attached to a four-to-five-residue amino-acid chain containing L-alanine, D-glutamine or D-isoglutamine, L-lysine and D-alanine (de Jonge et al., 1992, 1996). In the archaebacterial kingdom, methanogenic bacteria such as Methanopyrus kandleri replace murein with pseudomurein consisting of $N$-acetyl-D-galactosamine (GalNAc) and $N$-acetyl-Ltalosaminuronic acid (TalANAc) linked by a $\beta$-(1,3)-glycosidic bond and containing only L-amino acids such as L-alanine, L-lysine, L-glutamic acid and L-ornithine (Kurr et al., 1991).

Bacteria break down their murein components to synthesize the cell wall of the next generation (Goodell \& Schwarz, 1985). One of the steps involves anhydro- $N$-acetylmuramic acid kinase $($ AnmK), which converts 1,6-anhydro- $N$-acetylmuramic acid to $N$-acetylmuramic acid 6-phosphate with the simultaneous cleavage of the 1,6-anhydro ring and hydrolysis of ATP (Uehara et al., 2005). As the biosynthetic pathways of pseudomurein and murein differ (Hartmann \& König, 1990), no homologous enzymes or similar pathways for pseudomurein recycling have been identified in the genomes of microorganisms to date (Scheffers \& Pinho, 2005; Claus \& König, 2010). The methanogenic euryarchaeote M. kandleri
Received 25 April 2013

Accepted 12 August 2013

PDB References: MK0840 apo-m , 4bg8; MK0840 apo-o, 4bg9; MK0840-ADP ${ }^{\text {open }}$, 4bga; MK0840-ADP ${ }^{\text {closed }}, 4 \mathrm{bgb}$ 
Table 1

Data-collection and refinement statistics.

Values in parentheses are for the outermost shell.

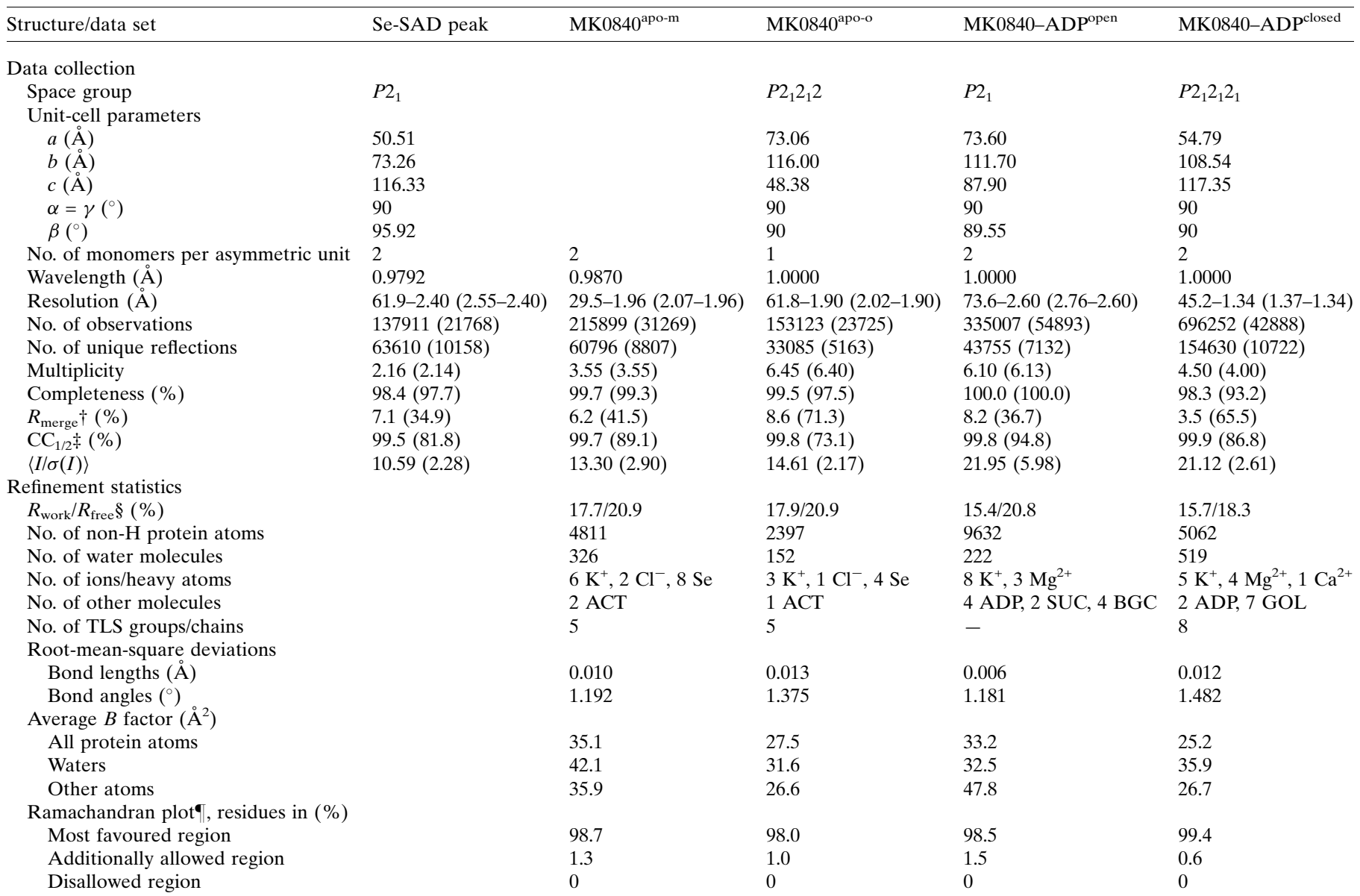

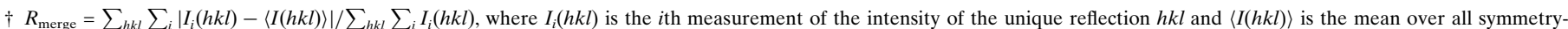

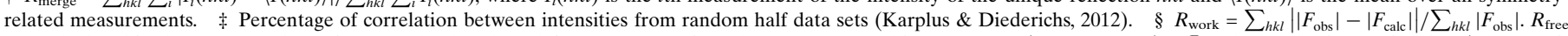

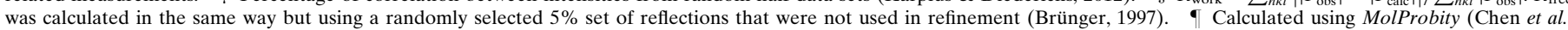
2010).

encodes a putative protein (UniProtKB entry Q8TX37; gene MK0840) that is similar to anhydro- $N$-acetylmuramic acid kinase and is annotated as a chaperone distantly related to Hsp70-fold metalloproteases. As archaebacteria lack $N$-acetylmuramic acid, replacing it with $N$-acetyltalosaminuronic acid, it is unlikely that they contain genes encoding the enzymes responsible for $\mathrm{N}$-acetylmuramic acid synthesis or degradation; rather, they would encode enzymes for the pathways involved in the metabolism of $\mathrm{N}$-acetyltalosaminuronic acid.

There is no structural information available for the enzymes of pseudomurein metabolic pathways. In order to gain a deeper insight into the mechanistic aspects, we have solved the crystal structure of MK0840 in different ligand-bound states and conformations and report the results here.

\section{Materials and methods}

\subsection{Cloning and expression}

A synthetic DNA fragment coding for the full-length putative sugar kinase from M. kandleri (MK0840, Q8TX37) and optimized for Escherichia coli expression (MrGene) was cloned into the pET28a vector (Novagen) with an N-terminal hexahistidine tag using the $N d e \mathrm{I} / X h o I$ restriction sites. Overexpression was carried out in E. coli BL21 (DE3) (Novagen) in LB medium overnight at $293 \mathrm{~K}$. Selenomethioninesubstituted protein was prepared using the metabolic inhibition method (Van Duyne et al., 1993; Doublié, 1997), yielding approximately the same amount of protein as the wild type. A truncated construct starting from amino acid Leu37 (Leu37Thr358) was cloned into the pET28a vector and overexpressed as described for the full-length construct.

\subsection{Purification}

Both full-length and truncated proteins were purified by immobilized metal-affinity chromatography (IMAC) using Ni-NTA Superflow resin (Qiagen) in a buffer consisting of $20 \mathrm{~m} M$ Tris- $\mathrm{HCl} \mathrm{pH} 7.5,300 \mathrm{~m} M \mathrm{NaCl}$ with $10-70 \mathrm{~m} M$ imidazole for the washing step or $250 \mathrm{~m} M$ imidazole for the elution steps. The hexahistidine tag was cleaved off by thrombin (Sigma-Aldrich) using $1 \mathrm{U}$ thrombin per milligram 
of protein during overnight dialysis against IMAC buffer lacking imidazole. The digested protein was again passed over an Ni-NTA column and subsequently further purified by sizeexclusion chromatography using a Superdex 200 16/60 column (GE Healthcare) and a buffer consisting of $20 \mathrm{~m} M$ Tris- $\mathrm{HCl}$ $\mathrm{pH} 7.5,200 \mathrm{~m} M \mathrm{NaCl}, 0.02 \%$ sodium azide and $4 \mathrm{~m} M$ DTT (or $10 \mathrm{~m} M$ DTT for selenomethionine-substituted protein). Fractions containing dimeric protein were pooled and were concentrated to $6 \mathrm{mg} \mathrm{ml}^{-1}$. The yield of pure protein was $6 \mathrm{mg}$ per litre of culture.

\subsection{Crystallization and data collection}

Initial crystallization screening was carried out using the sitting-drop vapour-diffusion method at $293 \mathrm{~K}$. As the apo crystals (MK0840 ${ }^{\text {apo }}$ ) obtained from full-length SeMetsubstituted MK0840 appeared overnight and were highly intergrown, several optimization procedures were carried out to slow down crystal growth. Finally, a very small number of crystals of diffraction quality could be obtained from sittingdrop experiments with $0.2 \%$ low-melting agarose (Hampton Research) and the initial condition $0.5 \mathrm{M}$ potassium thiocyanate, $0.1 M$ sodium acetate $\mathrm{pH} 4.6$ at $293 \mathrm{~K}$ with poor reproducibility. Small crystals growing at the very rim of the drop were seeded into the same drop by crushing with a thin needle. Only this procedure yielded thin and fragile plates with dimensions of $5 \times 50 \times 150 \mu \mathrm{m}$ and a single crystal lattice. For data collection, crystals were cryoprotected with the original mother-liquor solution supplemented with $40 \%$ sucrose (water replaced by sucrose) and were flash-cooled in liquid nitrogen. Diffraction data for selenium single-wavelength anomalous dispersion (Se-SAD) were collected under cryogenic conditions on beamline X06DA at the Swiss Light Source (PSI, Villigen, Switzerland) using a MAR225 detector. The wavelength for the Se-SAD peak was $0.9792 \AA$ and that for the remote was $0.9870 \AA$ (data set MK0840 $0^{\mathrm{apo}-\mathrm{m}}$; monoclinic space group). An additional data set from a second MK840 ${ }^{\text {apo }}$ crystal was collected to give higher resolution data (data set MK0840 ${ }^{\text {apo-o}}$; orthorhombic space group). Needleshaped crystals (MK0840-ADP ${ }^{\text {open }}$ ) with dimensions of $30 \times$ $30 \times 600 \mu \mathrm{m}$ were obtained from the N-terminally truncated construct (Leu37-Thr358) supplemented with $10 \mathrm{mM} \mathrm{MgCl}_{2}$ and $5 \mathrm{~m} M$ ADP (Sigma-Aldrich) in a condition consisting of $0.2 \mathrm{M}$ potassium sodium tartrate, $20 \%$ PEG 3350. Crystals were cryoprotected by soaking them in the original mother liquor supplemented with $20 \%$ sucrose for $30 \mathrm{~s}$ and were flashcooled in liquid nitrogen. Bar-shaped crystals (MK0840$\left.\mathrm{ADP}^{\text {closed }}\right)$ with dimensions of $40 \times 40 \times 200 \mu \mathrm{m}$ were also obtained for the N-terminally truncated construct (Leu37Thr358) supplemented with $10 \mathrm{mM} \mathrm{MgCl}_{2}, 75 \mathrm{mM} \mathrm{KCl}, 5 \mathrm{~m} M$ ADP (Sigma-Aldrich) in a cryocondition consisting of $0.14 \mathrm{M}$ $\mathrm{CaCl}_{2}, 0.07 \mathrm{M}$ sodium acetate $\mathrm{pH} 4.6,10 \%$ 2-propanol, $28 \%$ glycerol. Native data sets (data sets MK0840-ADP ${ }^{\text {open }}$ and MK0840-ADP ${ }^{\text {closed }}$ ) for molecular replacement were collected from both crystals at a wavelength of $1 \AA$ on beamline X06DA equipped with a MAR225 CCD detector. All data were indexed and processed using the $X D S$ software package (Kabsch, 2010). For MK0840-ADP ${ }^{\text {closed }}$ a lowresolution pass to $2.77 \AA$ resolution and a high-resolution pass to $1.34 \AA$ resolution were collected from the same crystal and were merged using XSCALE (Kabsch, 2010). The merged data set was used for structure determination and refinement. Data-collection and refinement statistics are summarized in Table 1.

\subsection{Structure determination and refinement}

The MK $0840^{\text {apo }}$ structure was solved by Se-SAD (Se-SAD peak data set) using phases computed from eight selenium sites by the phenix.autosol routine of the PHENIX package (Adams et al.,2010). The initial model was built with the ARP/ $w A R P$ web server (Morris et al., 2002; Langer et al., 2008) and was refined using iterative cycles of phenix.refine (Adams et al., 2010). Manual model building was accomplished using Coot (Emsley et al., 2010) using the remote data to $1.96 \AA$ resolution (data set MK0840 $0^{\text {apo-m }}$; space group $P 2_{1}$ ). For crossvalidation, a randomly selected subset $(5 \%)$ of reflections was set aside in order to monitor the progress of refinement using the free $R$ factor (Brünger, 1997). The coordinates were submitted to the TLS Motion Determination (TLSMD) server (Painter \& Merritt, 2006b) in order to resolve the optimal number of TLS groups for subsequent use in TLS refinement (Painter \& Merritt, 2006a) with phenix.refine. Data from a second apo crystal (MK0840 apo-o; space group $\left.P 2_{1} 2_{1} 2\right)$ grown in the same drop as the $P 2_{1}$ SeMet crystal (MK0840 ${ }^{\text {apo-m }}$ ) could be collected to $1.9 \AA$ resolution. The structure was solved by molecular replacement using the program Phaser (McCoy et al., 2007) in the CCP4 program suite (Winn et al., 2011) with one monomer of MK $0840^{\text {apo-m }}$ as the search model. For both apo structures, anomalous scattering corrections $\left(f^{\prime} / f^{\prime \prime}\right)$ for the Se atoms were included during refinement.

The MK0840-ADP ${ }^{\text {open }}$ and MK0840-ADP ${ }^{\text {closed }}$ structures were determined by molecular replacement with Phaser using one monomer of the refined MK0840 $0^{\text {apo-m }}$ structure as a search model for MK0840-ADP ${ }^{\text {open }}$ and one monomer of MK0840$\mathrm{ADP}^{\text {open }}$ for MK0840-ADP ${ }^{\text {closed }}$. As one of the two protomers in the MK0840-ADP ${ }^{\text {closed }}$ structure differed substantially from the model used, only a partial solution was found. The missing protomer was built by $A R P / w A R P$. The nucleotide-bound structures were refined as described for MK0840 $0^{\text {apo-m }}$, but for MK0840-ADP ${ }^{\text {open }}$ TLS refinement did not improve the model as judged by an increase in $R_{\text {free }}$ and a larger gap between $R_{\text {work }}$ and $R_{\text {free }}$

The final structures were validated using MolProbity (Chen et al., 2010), WHATCHECK (Vriend, 1990) and the corresponding tools in Coot. Secondary-structure elements were assigned using DSSP (Kabsch \& Sander, 1983). Structural alignment was performed with STRAP (Gille \& Frömmel, 2001), sequence alignment was performed using ClustalW2 (Larkin et al., 2007) and alignment visualization was performed using ESPript (Gouet et al., 1999). Figures were prepared with PyMOL (DeLano, 2002) and LigPlot $^{+}$v.1.4.2 (Laskowski \& Swindells, 2011). 


\subsection{Substrate modelling}

Clear densities for ADP and additional ligands bound to the putative sugar-binding pocket were found during refinement of the MK0840-ADP ${ }^{\text {open }}$ and MK0840-ADP ${ }^{\text {closed }}$ structures. ADP molecules were modelled into the density using phenix.ligandfit (Terwilliger et al., 2007). A ligand search was performed using electron-density map correlation with the program phenix.ligand_identification (Terwilliger et al., 2006) to identify the molecule occupying the substrate-binding pocket of MK0840-ADP ${ }^{\text {open }}$. The molecules with the best correlation coefficients $(\mathrm{CC})$ were BGC $(\beta$-D-glucose; CC $=$ $0.73)$, AMG $(\alpha$-methyl-D-galactoside; $\mathrm{CC}=0.71)$ and GAL $(\beta$-D-galactose; $\mathrm{CC}=0.70)$. The electron density in the substrate-binding pocket was inspected and $\beta$-D-glucose was chosen. The sucrose in MK0840-ADP ${ }^{\text {open }}$ and the glycerol molecules in MK0840-ADP ${ }^{\text {closed }}$ were manually fitted into the electron density. Simulated-annealing $2 F_{\mathrm{o}}-F_{\mathrm{c}}$ OMIT maps were calculated using the RESOLVE routine of phenix.autobuild (Terwilliger et al., 2008).

\subsection{Data deposition}

The atomic coordinates and structure factors have been deposited in the Protein Data Bank (Berman et al., 2000; http://www.pdb.org) with PDB codes 4 bg8 for MK0840 apo-m, 4bg9 for MK0840 apo-o, 4bga for MK0840-ADP ${ }^{\text {open }}$ and 4bgb for MK0840-ADP ${ }^{\text {closed }}$ ).

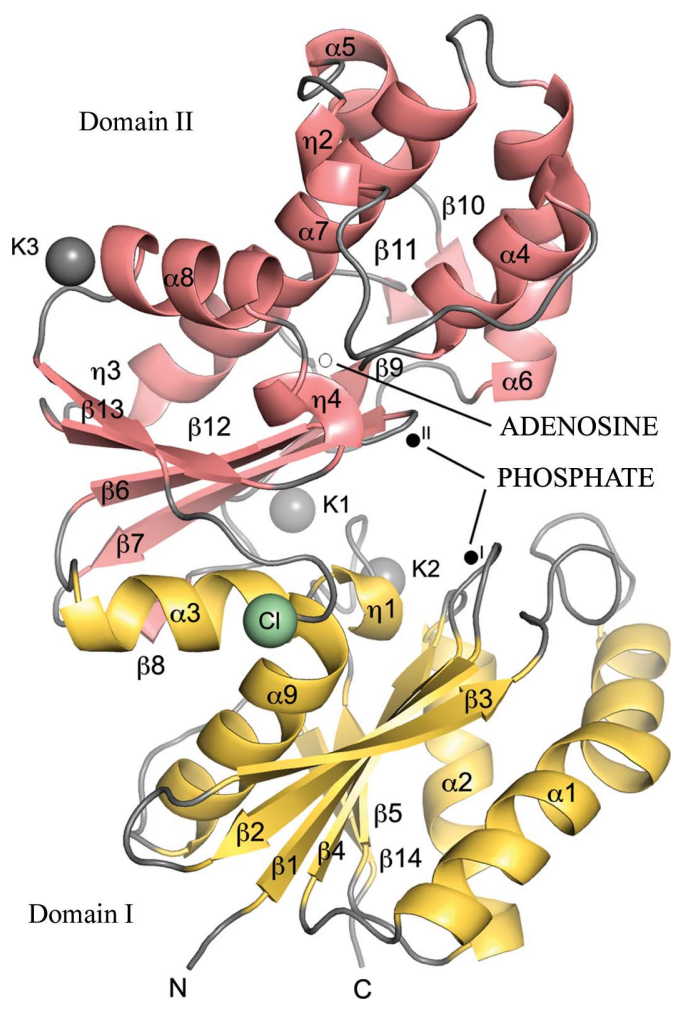

Figure 1

Overall structure of the MK0840 ${ }^{\text {apo }}$ monomer. The N-terminal domain I is shown in yellow and the C-terminal domain II in light pink. The potassium ions (K1-K3) and the chloride ion $(\mathrm{Cl})$ are shown as spheres. The adenosine and phosphate-binding regions (I and II) are indicated by open and solid circles, respectively.

\section{Results and discussion}

The following crystal structures were determined in this study: MK0840 without bound nucleotide (termed MK0840 $0^{\text {apo }}$ ) in two space groups and ADP-bound open (MK0840-ADP ${ }^{\text {open }}$ ) and closed (MK0840-ADP ${ }^{\text {closed }}$ ) forms. The structures were refined at diffraction limits of $2.60-1.34 \AA$ with satisfactory statistics (Table 1).

\subsection{Monomer structure}

A database search for similar folds resulted in Pseudomonas aeruginosa 1,6-anhydro- $N$-acetylmuramic acid kinase (PaAnmK; PDB entry 3qbw; Bacik et al., 2011) and Acidaminococcus fermentans 2-hydroxyglutaryl-CoA dehydratase component A (HgdC; PDB entry 1hux; Locher et al., 2001) as top hits as well as an uncharacterized protein from Shewanella oneidensis (SoAnmK; PDB entry 3cqy; Midwest Center for Structural Genomics, unpublished work). Sequence identities for all of these matches were below $20 \%$ and the r.m.s. deviations of the superposed structures were $3 \AA$ or greater for equivalent $\mathrm{C}^{\alpha}$ atoms. Thus, the overall structure of the M. kandleri MK0840 monomer shows a typical ribonuclease H-like type ASKHA superfamily sugar kinase fold (Bork et al., 1992) consisting of two $\alpha / \beta$ subdomains related to each other by approximate dyad symmetry (Fig. 1). Domain I comprises residues Leu37-Pro159 as well as Gly322-Thr358 and domain II contains residues Asn160-Ala321. The topology of domain I is of the form $\beta \beta \beta \alpha \beta \alpha \beta \alpha$ with the $\beta$-strands in the order 3-2-1-4-5 with strand $\beta 2$ antiparallel to the others, with the very $\mathrm{C}$-terminal strand $\beta 14$ completing the $\beta$-sheet in an antiparallel manner, and helices $\alpha 1, \alpha 2$ and $\eta 1 / \alpha 3$ (where $\eta$ represents a $3_{10}$-helix) as well as helix $\alpha 9$.

Domain II contains strands $\beta 6-\beta 13$ in the order $9 / 8-7-6-$ $12-13$, with $\beta 7$ antiparallel to the others, and $\beta 10-\beta 11$ as well as helices $\alpha 4-\alpha 8$ and $\eta 2-\eta 4$. The ATP-phosphate binding regions in the kinase fold were located in domain II $\left(\mathrm{L}^{45} \mathrm{GNT}^{48}\right.$ and PHOSPHATE I; $\mathrm{V}^{164} \mathrm{DVGA}^{168}$ and PHOSPHATE II) as well as the adenosine-binding region $\left(\mathrm{S}^{296}\right.$ GGGVKN $^{302}$ and ADENOSINE) on helix $\eta 4$ (Fig. 1 and Supplementary Fig. S1 ${ }^{\mathbf{1}}$ ); pattern identification is according to Bork et al. (1992).

MK0840-ADP ${ }^{\text {open }}$ binds sucrose molecules originating from the cryoprotectant solution at the contact interface of protomers $A$ and $C$ as well as that of protomers $B$ and $D$ (Fig. 3c). The residues involved in sucrose coordination are located on strand $\beta 3$ of both protomers. Several hydrogenbonded and nonbonded interactions are formed to hydroxyl groups of both the hexose and pentose of the sucrose.

All protomers in both of the MK0840 ${ }^{\text {apo }}$ structures and the MK0840-ADP ${ }^{\text {closed }}$ structure and three of the four protomers in the MK0840-ADP ${ }^{\text {open }}$ structure lack electron density for the eight very $\mathrm{C}$-terminal residues in the polypeptide chain. Only for one protomer of MK0840-ADP ${ }^{\text {open }}$ could the flexible C-terminus be modelled into the electron-density map owing

\footnotetext{
${ }^{1}$ Supplementary material has been deposited in the IUCr electronic archive (Reference: GM5025). Services for accessing this material are described at the back of the journal.
} 
to its involvement in crystal contacts. The stretch between Gly351 and Thr358 contains four glycine residues, which probably makes it highly flexible in solution. During refinement and model building we were initially not able to locate the first 36 amino acids of full-length MK0840 in the electron density, indicating that these residues are disordered in the crystal. Secondary-structure and tertiary-structure prediction tools (Yang et al., 2005; Slabinski et al., 2007; Kelley \& Sternberg, 2009), on the other hand, predicted an ordered $\mathrm{N}$-terminus with residues Ala19-Thr31 in an $\alpha$-helical conformation. By closer inspection of the electron density, residues Val23-Thr36 could be modelled into uninterpreted electron density. This part of the structure clearly displays an $\alpha$-helical conformation, but the remaining part of the $\mathrm{N}$-terminus is still missing. Interestingly, these first 36 residues are absent in other ASKHA family kinases (Supplementary Fig. S1). The function of the first 36 amino acids of MK0840 is unclear, but they seem to be an archaebacterial feature.

\subsection{Ion-binding sites}

During refinement, three potential cation-binding sites per monomer were identified in the MK0840 $0^{\text {apo }}$ structure. Based on the type and the arrangement of the coordinating ligands (carbonyl $\mathrm{O}$ atoms and carboxylate groups of aspartate or glutamate residues as well as water molecules) and the observed distances (average $2.95 \AA$ ), $\mathrm{K}^{+}$was selected as the most likely cation. The refined $B$ factors of the potassium ions match those of the surrounding protein atoms, indicating full occupancy. Furthermore, for all assigned $\mathrm{K}$ atoms anomalous difference signals were detected, which lends further credence to the assignment of these metal ions as potassium. As all crystallization conditions contained potassium salts, the bound $\mathrm{K}^{+}$ions most probably originate from the mother liquor.

Two of the three binding sites are present in all crystal forms. They are close to each other, with the ions (K1 and
K2) being $7.8 \AA$ apart and 'sandwiched' between three loop structures to the left and right of Trp140 (Fig. 2a), a residue that plays a role in dimer formation. The two potassium ions are located near the substrate-binding site, with $\mathrm{K} 2$ being approximately $7 \AA$ away from the $\mathrm{O} 2$ atom of $\beta$-D-glucose in MK0840-ADP ${ }^{\text {open }}$ and $6 \AA$ away from the $\mathrm{O} 3$ atom of glycerol in MK0840-ADP ${ }^{\text {closed }}$. Strand $\beta 8$ is interrupted at Pro180 and continues as $\beta 9$ at Val186. Between them, the polypeptide chain (Asp181-Ala185) performs a turn in order to allow the coordination of the potassium ion $\mathrm{K} 1$, a feature that is not found in the structures of other ASKHA members (e.g. E. coli RhuK). There is a cis-peptide bond between Ala142 and Pro143. Both residues are located in the loop region between strand $\beta 5$ and helix $\eta 1$ and are involved in the coordination of potassium ions $\mathrm{K} 1$ and $\mathrm{K} 2$ (Fig. 2a). The cis-peptide bond is probably formed to allow bending of the polypeptide chain around K2. Residues Pro143 and Gly141 bind K2 with their backbone $\mathrm{O}$ atoms enclosing an angle of $82^{\circ}$. This is only made possible by the cis conformation of the Ala-Pro peptide bond. cis-Peptide bonds are known to occur in turns and near ionbinding sites (Pal \& Chakrabarti, 1999).

In the two MK0840 apo structures the third potassiumbinding site belongs to the $440 \AA^{2}$ contact interface between one monomer and a symmetry-equivalent monomer from another biological dimer (Fig. $3 b$ ) as determined by the PISA server (Krissinel \& Henrick, 2007). Contrary to those of K1 and $\mathrm{K} 2$, this site appears to be generated by the crystal lattice and therefore may not be occupied in solution. An acetate ion is bound close to $\mathrm{K} 3$ which originates from the crystallization solution and interacts with the terminal amino groups $\mathrm{N}^{\eta 2}$ of Arg307 and $\mathrm{N}^{\eta 1}$ of Arg308 via its carboxyl group as well as with Glu311 via a hydrogen bond (Fig. 3b).

As mentioned above, the observed ions probably originate from the crystallization buffers and it is not known whether potassium is bound to the enzyme in the cellular environment. However, this appears to be likely owing to the full occupancy

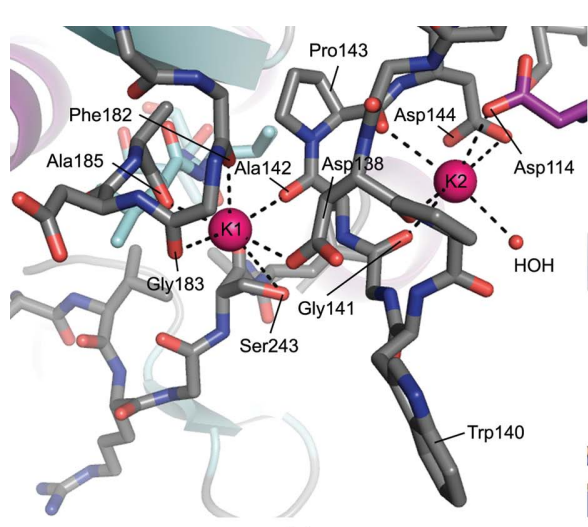

(a)

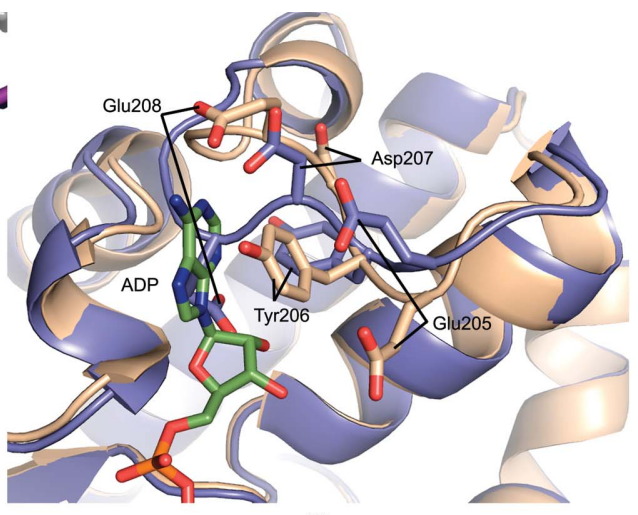

(b)

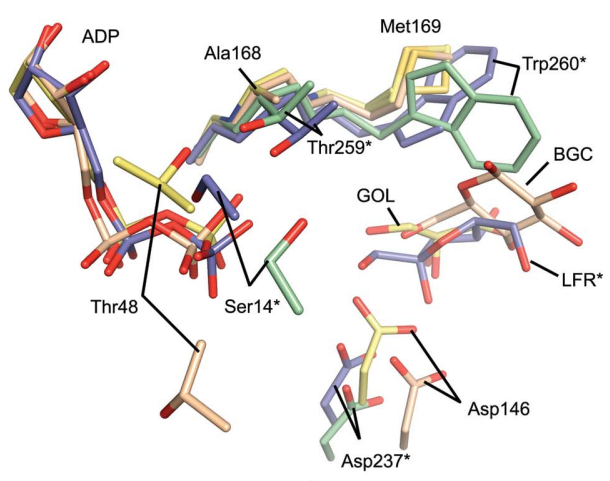

$(c)$

Figure 2

Structural features of MK0840. (a) Potassium-binding sites K1 and K2 observed in both the MK0840 apo and the MK0840-ADP structures. The polypeptide chain is shown in stick representation. Potassium ions are depicted as pink spheres. Bonds to the potassium ions are shown as dashed black lines. (b) Superimposition of the nucleotide-binding region of $\mathrm{MK} 0840^{\text {apo }}$ (blue) and MK0840-ADP ${ }^{\text {open }}$ (wheat). The ADP molecule and important side chains are shown in stick representation. (c) Superimposition of MK0840-ADP ${ }^{\text {open }}$ (wheat), MK0840-ADP ${ }^{\text {closed }}$ (yellow) and rhamnulose kinase RhuK in the apo state (green; PDB entry 2cgk) and with bound ADP and fructose (blue; PDB entry 2cgj) in the nucleotide-binding and substrate-binding region. Important side chains are shown in stick representation and asterisks indicates residues of RhuK. The carbohydrates $\beta$-L-fructose (LFR), $\beta$-Dglucose (BGC) and glycerol (GOL) are shown in stick representation. $\mathrm{O}$ atoms are coloured red, $\mathrm{N}$ atoms dark blue and $\mathrm{S}$ atoms yellow. 
together with the general high intracellular potassium ion concentrations. Potassium ions have been reported to be important for the thermostability and activity of proteins in thermophilic archaea (Breitung et al., 1992). It has been shown for proteins from $M$. kandleri that enzyme activity and stability at high temperatures are promoted by high concentrations of potassium salts of cyclic 2,3-diphosphoglycerate, 2,3-bisphosphoglycerate (both at $0.75 \mathrm{M}$ ) or phosphate (at 1.5 M) (Shima et al., 1998; Mamat et al., 2002). This requirement correlates with high intracellular concentrations of cyclic 2,3-diphosphoglycerate $(\sim 1 M)$ in hyperthermophilic M. kandleri (Shima et al., 1998).

Additionally to these potassium ions, MK0840 apo also binds an anion, which was identified as chloride (Fig. 1). It is coordinated in a pyramidal geometry by Glu324 N, Arg328 N ${ }^{\eta 1}$ and a water molecule. The anion-ligand distances are 2.9$3.4 \AA$ and are in the range observed for other chloride-binding sites (Qian et al., 1994; Feller et al., 1996).

\subsection{Dimer formation}

In solution, full-length and truncated M. kandleri MK0840 forms dimers and tetramers in a 3:1 ratio, as observed in size- exclusion chromatography and native PAGE experiments. However, only the dimeric protein produces crystals. In the MK0840 apo-m crystal form belonging to space group $P 2_{1}$, the asymmetric unit contains a dimer with the two monomers related by a twofold noncrystallographic symmetry axis. This dimer is observed in all of the crystal forms reported here and is unusual. Most dimeric ASKHA family members dimerize via their C-terminal domains II (Lunin et al., 2004; Mukai et al., 2004; Gorrell et al., 2005; Simanshu et al., 2005; Hong et al., 2006; Nishimasu et al., 2007), burying a surface area of approximately 1400-3100 $\AA^{2}$ per monomer. In contrast, MK0840 forms a dimer via the N-terminal domains I of each monomer (Fig. $3 a$ and Supplementary Fig. S4), with a surface area of $1455 \AA^{2}$ per monomer in MK0840 apo-m as determined using the PISA server (Krissinel \& Henrick, 2007). The 40 residues involved from each monomer interact by 13 hydrogen bonds and five salt bridges. A similar dimer formation via domains I is only observed in SoAnmK (PDB entry 3cqy; Midwest Center for Structural Genomics, unpublished work), involving 51 residues and a surface area of $1844 \AA^{2}$ per monomer, as well as in glycerol kinase from Enterococcus casseliflavus (Yeh et al., 2003), involving 44 residues and an area of $1400 \AA^{2}$ per monomer. In the contact region between
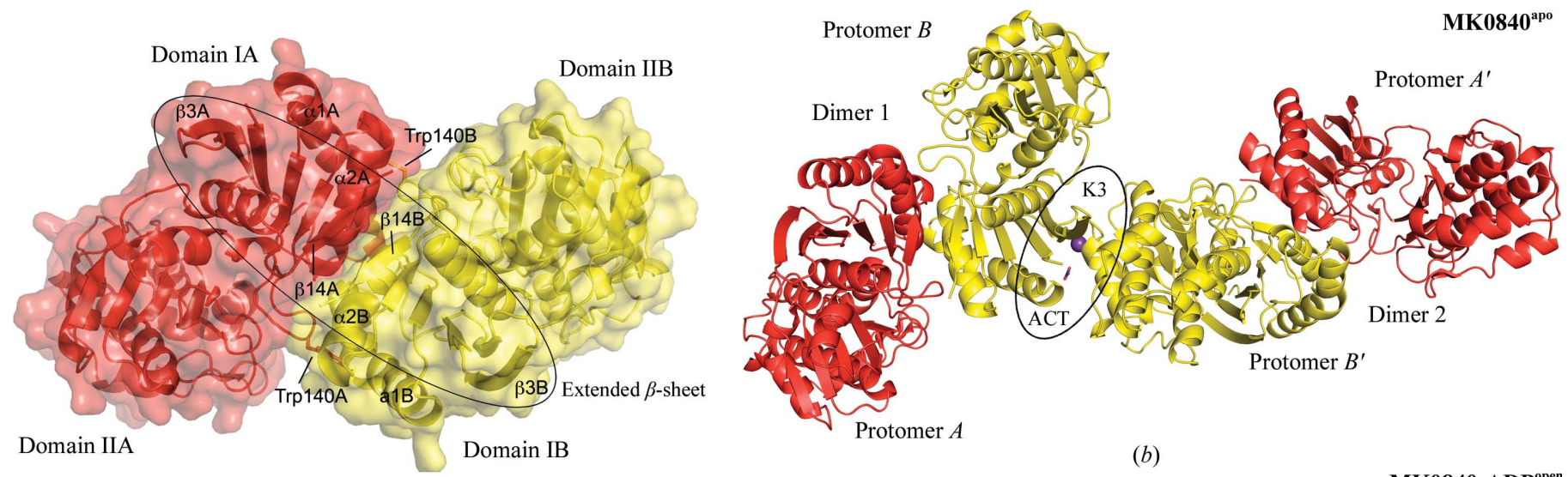

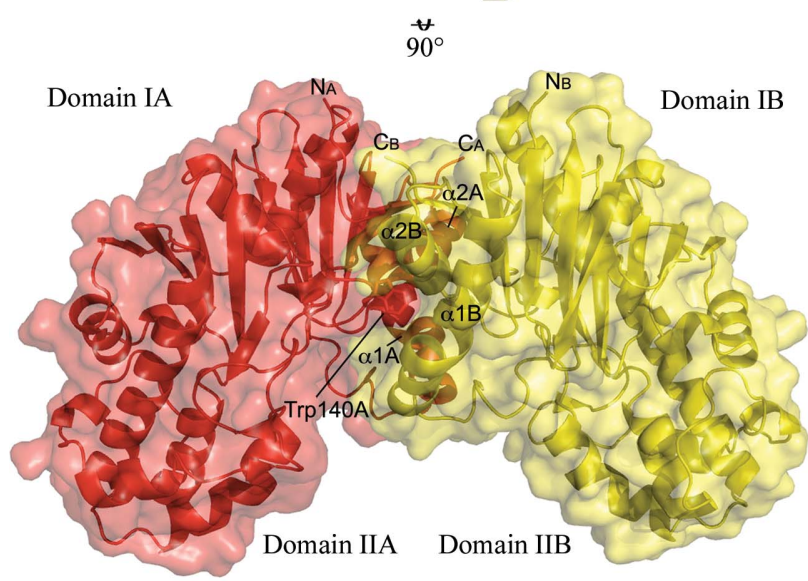

(a)

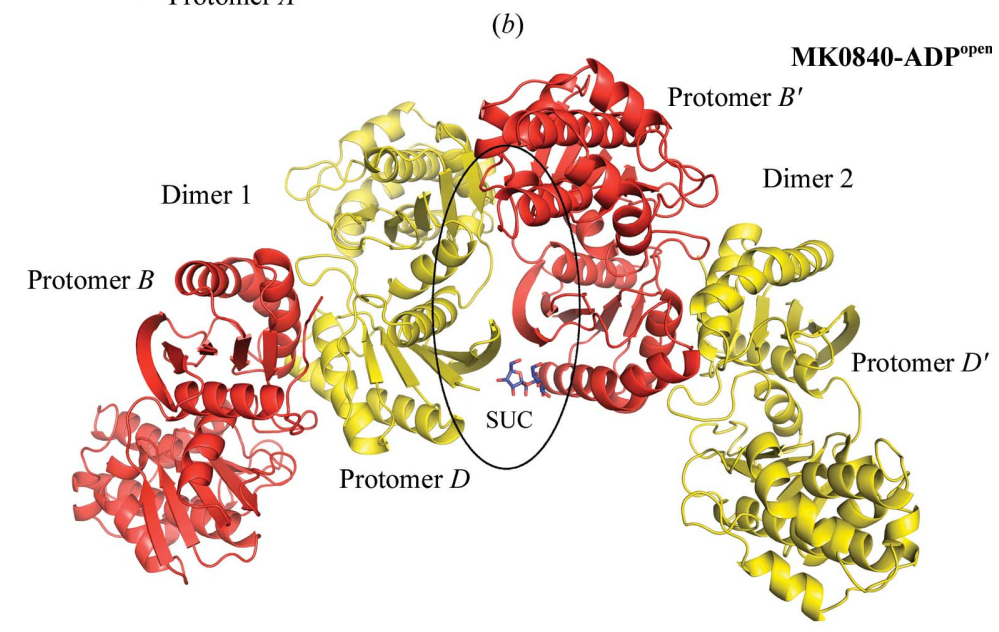

(c)

Figure 3

Dimer formation in MK0840. (a) Both MK0840 apo and MK0840-ADP form a dimer involving both domains I building up an extended $\beta$-sheet. Protomers $A$ and $B$ are coloured red and yellow, respectively. The molecular surface and structural elements involved in dimer formation are also shown. MK0840 ${ }^{\text {apo }}(b)$ and MK0840-ADP ${ }^{\text {open }}(c)$ build up additional contact interfaces that differ from each other. $(b)$ MK0840 ${ }^{\text {apo }}$ buries an acetate (ACT) molecule and a potassium ion (K3) within the crystallographic contact interface between protomers $B$ and $B^{\prime}$ from two distinct dimers. (c) In MK0840$\mathrm{ADP}^{\mathrm{open}}$ the crystallographic contact interface between protomers $D$ and $B^{\prime}$ from two distinct dimers buries the sucrose (SUC) molecule. 
the two monomers of MK0840 an extended $\beta$-interface is formed by the interplay of $\beta$-strands 14 (Leu346-Cys348) on both protomers, building up a continuous sheet spanning the two subunits (Fig. $3 a$ and Supplementary Fig. S4). Most of the contact area is formed by interactions between residues located in helices $\alpha 1$ and $\alpha 2$. Each monomer provides Trp140, a residue located in a tight turn between strand $\beta 5$ and helix $\eta 1$ and which extends into the other monomer, contacting residues Arg88, Ala92, Ile96, Glu126 and Lys127 via van der Waals interactions, and Met123 via a hydrogen bond.

\subsection{Nucleotide-binding site and domain closure}

In order to investigate nucleotide binding and potential accompanying conformational rearrangements, we undertook cocrystallization of the N-terminally truncated construct with ADP. Two different crystal forms were obtained under different conditions, termed $\mathrm{ADP}^{\text {open }}$ and $\mathrm{ADP}^{\text {closed }}$. They differ in ligand state, crystal packing and the conformation of the MK0840 molecules, but have the following features in common.

Both MK0840-ADP structures have ADP bound (Fig. 4 and Supplementary Fig. S5). While both apo crystal forms display a less well defined stretch between Glu205 and Lys211 (Fig. 2b), indicating flexibility in this part of the structure, this segment becomes more ordered upon nucleotide binding. Several side chains have to move to allow the binding of the adenine moiety of the nucleotide. The largest changes are observed in the $\chi_{1}$ angles of Glu205, which changes from 87 to $-148^{\circ}$ (from apo to ADP-bound), and of Tyr206, which flips from -178 to $54^{\circ}$. By adopting the new position in the MK0840-ADP structures, the phenol ring of Tyr206 can engage in $\pi-\pi$ stacking interactions with the adenine moiety of ADP. In the apo structures Glu208 occupies the spatial position of the adenosine part of ADP (Fig. 2b), whereas in the MK0840-ADP structures the adenine N6 atom is coordinated by $\mathrm{O}^{\varepsilon 1}$ of this residue (Figs. $2 b, 4 a$ and $4 b$ ).
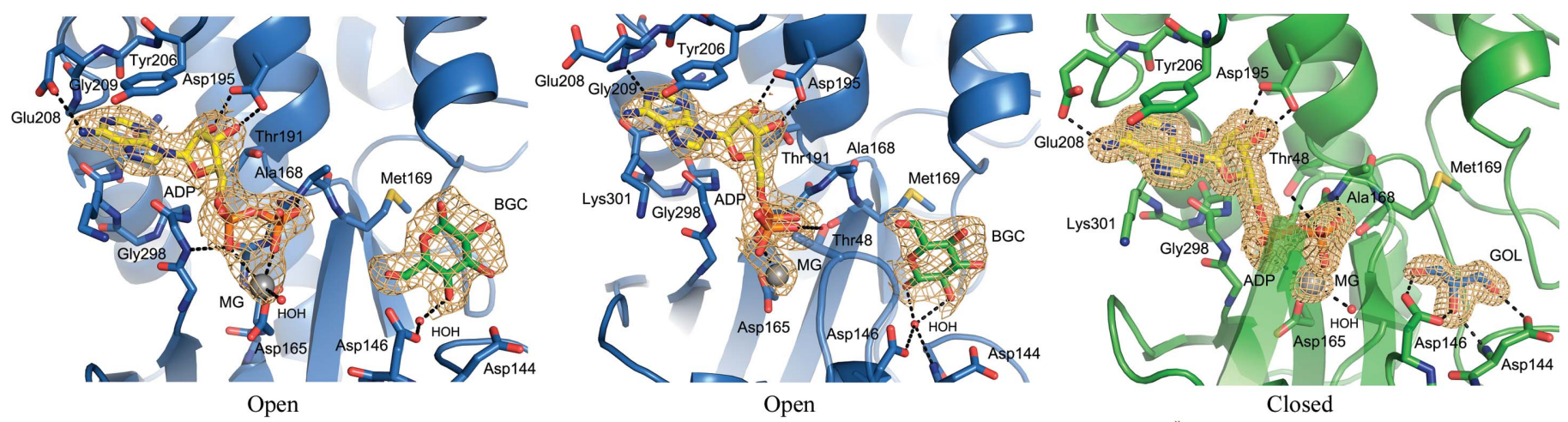

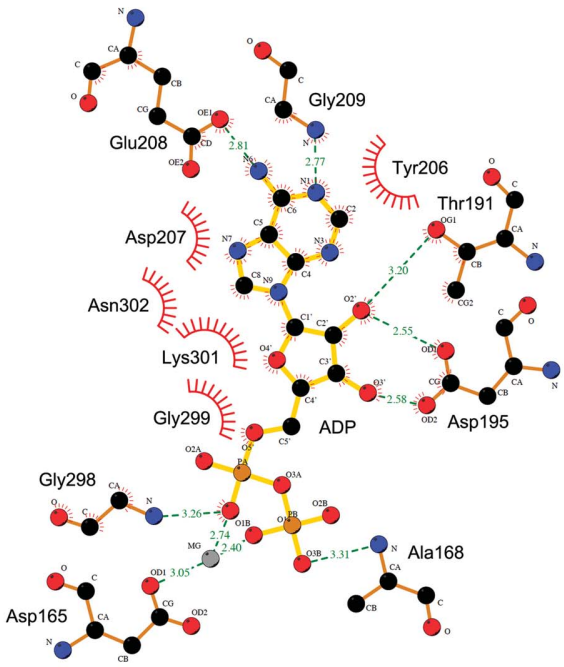

(a)

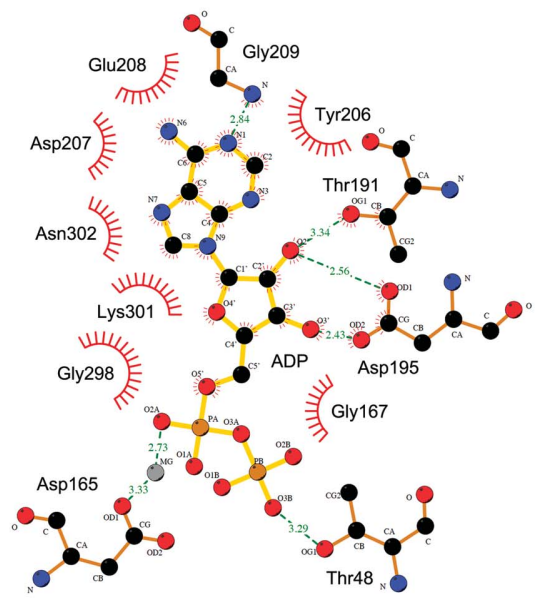

(b)

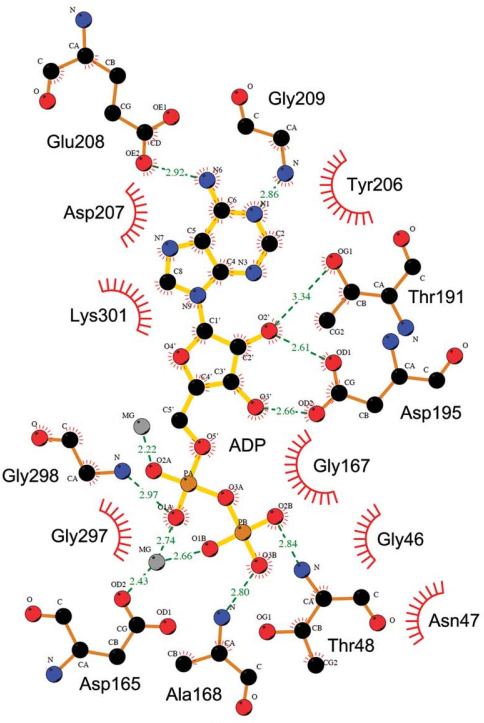

(c)

Figure 4

Nucleotide and substrate binding differs in the open and closed forms of MK0840. In protomers $B$ and $D$ of MK0840-ADP ${ }^{\text {open }}$ the $\beta$-phosphate of ADP points towards the $\beta$-D-glucose (BGC) molecule ( $a$ ), while in protomers $A$ and $C$ it points towards Thr48 $(b)$. The set of residues coordinating the adenine and ribose moiety also differs. In protomers $A$ and $C(b)$ interactions with Ala168 and Glu208 are missing but a new interaction with Thr48 is formed. (c) In MK0840-ADP ${ }^{\text {closed }}$ glycerol (GOL) is bound. The ADP molecule in $(c)$ adopts the same conformation as in $(a)$ but interacts simultaneously with Ala168 and Thr48, leading to domain closure. In all six protomers of both MK0840-ADP forms the adenine moiety of ADP is stacked against the phenol ring of Tyr206 and the hydroxyl groups of the ribose are coordinated by Asp195. For better visibility the loop containing Thr48 was removed in $(a)$. In (a) $-(c) 2 F_{\mathrm{o}}-F_{\mathrm{c}}$ OMIT electron-density maps (light orange) of the ADP molecule, the $\mathrm{Mg}^{2+}$ ion, BCG $(a, b)$ and GOL $(c)$ contoured at $1.0 \sigma$ are shown (upper row). The ligand plots depict interactions of the ADP molecule with protein residues (lower row). Green dashes represent the hydrogen-bonding network; numbers represent distances in $\AA$. 
The ADP molecule binds in the cleft of domain II formed by residues located on helix $\alpha 4$ and the loop connecting it to the $3_{10}$-helix $\eta 2$ as well as on helix $\eta 4$. The ribose ring is in the $\mathrm{C} 3^{\prime}$-endo conformation, with the $2^{\prime}$-oxygen and the $3^{\prime}$-oxygen forming hydrogen bonds to the side-chain carboxyl group of Asp195 and van der Waals interactions with the hydroxyl group of Thr191. The adenine ring adopts the anti conformation and is sandwiched between the side chains of Tyr206 and Lys301 (Fig. 4 and Supplementary Fig. S5). The adenosine moiety makes additional van der Waals interactions with the backbone of residues in helix $\eta 4$ as well as water-mediated hydrogen bonds with the backbone amide of Asp207 and the carboxamide side chain of Asn302. The $\alpha$-phosphoryl group of ADP interacts with the backbone amide of Gly298 and a $\mathrm{Mg}^{2+}$ ion, which itself is coordinated by the carboxyl group of Asp165.

The two crystal forms exhibit distinct differences that will be described in the following.

3.4.1. The MK0840-ADP ${ }^{\text {open }}$ crystal form. These crystals belonged to the monoclinic space group $P 2_{1}$ and contained four molecules per asymmetric unit. All of them exhibit an open conformation very much resembling the apo forms observed in the non-ligand-bound structures MK0840 apo-o and MK $0840^{\text {apo-m }}$. The r.m.s.d. between protomers $A$ of the MK0840 $0^{\text {apo-m }}$ structure and the MK0840-ADP ${ }^{\text {open }}$ structure is $0.65 \AA$ for $313 \mathrm{C}^{\alpha}$ atoms, indicating that ADP binds to the enzyme without causing major changes in domain orientation. It is known for ASKHA family members that domain closure can occur upon substrate or nucleotide binding (Yeh et al., 2003; Grueninger \& Schulz, 2006; Hong et al., 2006). For proteins such as Hsc70 or actin, which use MgATP or magnesium polyphosphate to carry out their function, the nucleotides alone are able to induce domain closure (Frieden et al., 1980; Wilbanks \& McKay, 1995). However, for kinases which bind MgATP and additionally specific smallmolecule substrates, e.g. carbohydrates, the substrate alone is sufficient to close the cleft (Buss et al., 2001; Nishimasu et al., 2007). The sole binding of ADP or other nucleotides usually leads to open conformations (Simanshu et al., 2005; Schnick et al., 2009). Both the MK0840 apo and the MK0840-ADP ${ }^{\text {open }}$ structures resemble the open conformations observed in other ASHKA kinases, e.g. SoAnmK, in which a succinic acid molecule is bound (PDB entry 3cqy; Midwest Center for Structural Genomics, unpublished work), or E. coli RhuK, in which ADP and L-fructose were found in the crystal structure (Grueninger \& Schulz, 2006).

During refinement, we could observe differences in the conformation and

Figure 5 coordination of the ADP molecules in the individual protomers, in which the $\beta$-phosphate groups can occupy alternative positions (Figs. $4 a$ and $4 b$ and Supplementary Figs. S5a and $\mathrm{S} 5 b)$. Protomers $B$ and $D$ show torsion angles $\left(\mathrm{C}^{\prime}-\mathrm{O}^{\prime}-\right.$ PA $-\mathrm{O} 3 \mathrm{~A})$ of -60 and $-28^{\circ}$, respectively, whereas protomers $A$ and $C$ exhibit angles of 64 and $30^{\circ}$, respectively. These torsion angles lie in the range observed in the structures of other ASKHA family kinases, e.g. -50 and $-66^{\circ}$ for $P a A n m K$ (PDB entry 3qbw; Bacik et al., 2011) and $67^{\circ}$ (for both protomers) for acetate kinase (PDB entry 1tuy; Gorrell et al., $2005)$. Consequently, the $\beta$-phosphate ( $\mathrm{P} \beta$ ) groups point in different directions: in protomers $B$ and $D$ the $\mathrm{P} \beta$ is mainly located nearer to the putative substrate-binding site, simultaneously interacting with the backbone amide of Ala168 located in the turn between strands $\beta 6$ and $\beta 7$ in domain II (Fig. $4 a$ and Supplementary Fig. S5a). In protomers $A$ and $C$ the $\beta$-phosphate group points towards Thr48, a residue that is located in the turn connecting strands $\beta 1$ and $\beta 2$ in domain I, and interacts with its hydroxyl group (Fig. $4 b$ and Supplementary Fig. S5b). Superimposition of MK0840-ADP ${ }^{\text {open }}$ with other kinases such as rhamnulose kinase (RhuK; Fig. 2c), $P$. aeruginosa panthothenate kinase $(\mathrm{PaCoA})$ and the 2-hydroxyglutaryl-CoA dehydratase component A ( $\mathrm{HgdC})$ from $A$. fermentans reveals that $\mathrm{Thr} 48$ occupies the same spatial position as serine or threonine residues in these enzymes. These residues are involved in binding to the $\beta$-phosphate via their backbone amides and/or their side-chain hydroxyls (Ser14 in RhuK, Thr10 in $\mathrm{PaCoaA}$ and Ser12-Thr13 in $\mathrm{HgdC}$ ). They belong to the conserved PHOSPHATE I-

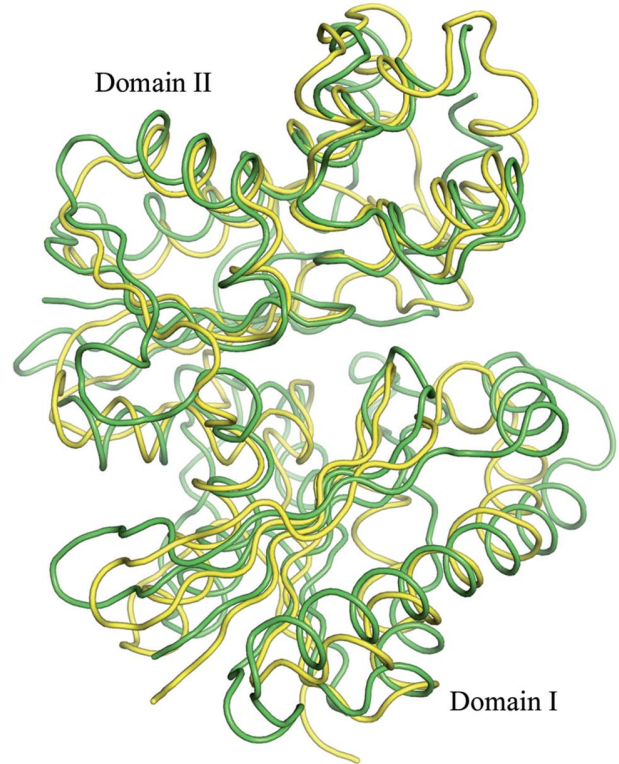

(a)

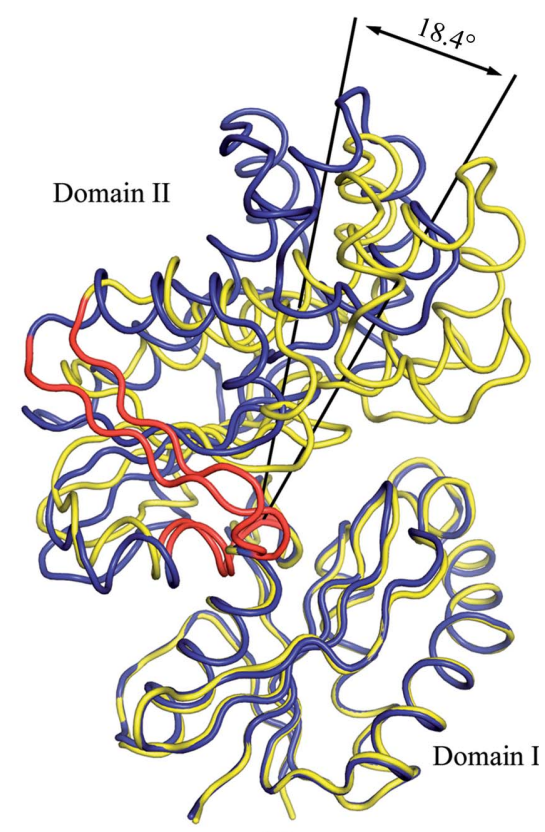

(b)

Domain closure in MK0840. (a) Superimposition of the closed conformation of MK0840-ADP closed $^{\text {. }}$ (yellow) with the closed conformation of $P a A n m K$ (green; PDB entry 3qbx). (b) Superimposition of the closed conformation of MK0840-ADP ${ }^{\text {closed }}$ (yellow) with the open conformation of MK0840$\mathrm{ADP}^{\text {closed }}$ (blue). The degree of domain closure is indicated by arrows. The bending region is coloured red. A tube representation of the protein backbone is shown. 
binding region in the ASKHA superfamily (Fig. 1 and Supplementary Fig. S1).

3.4.2. The MK0840-ADP ${ }^{\text {closed }}$ crystal form. This crystal form belonged to the orthorhombic space group $P 2_{1} 2_{1} 2_{1}$ and contained two crystallographically independent molecules. Protomer $A$ exhibits a conformation very similar to the apo and $\mathrm{ADP}^{\text {open }}$ crystal forms. The coordination pattern of the bound $\mathrm{ADP}$ is similar to that of $\mathrm{ADP}^{\text {open }}$, including alternating positions of the $\beta$-phosphate group. However, for protomer $B$ of MK0840-ADP ${ }^{\text {closed }}$ a closed conformation could be assigned similar to the conformation of ADP-bound $\mathrm{PaAnmK}$ (Fig. 5a). Compared with the open conformation of MK0840 ${ }^{\text {open }}$, in the closed conformation domain II makes an $18.4^{\circ}$ movement towards domain I (Fig. $5 b$ ) as calculated by the DynDom server (Hayward \& Berendsen, 1998). The typical angle of domain movement in ASKHA family kinases lies in the range $10-30^{\circ}$, e.g. $14.9^{\circ}$ for glycerol kinase [open, PDB entry 2p3r $(E)$; closed, PDB entry 2p3r $(A)], 18.3^{\circ}$ for hexokinase [open, PDB entry 2e2n $(A)$; closed, PDB entry 2e2q $(A)$ ] or $29.4^{\circ}$ for 3-phosphoglycerate kinase [open, PDB entry 1qpg $(A)$; closed, PDB entry 13 pk $(A)$ ], all taken from the DynDom database of specific domain movements (Qi \& Hayward, 2009). The bending regions in protomer $B$ of MK0840-ADP ${ }^{\text {closed }}$ were identified as Asp146-Pro151 (helices $\eta 1$ and $\alpha 3$ ) and Glu314-Glu324 (strand $\beta 13$ ), which are all residues connecting domains I and II. They fall into the conserved motifs CONNECT I and CONNECT II, respectively, as identified by Bork et al. (1992), that have been demonstrated to serve as the interdomain hinge in ASKHA proteins. Interestingly, the residue Asp146 that is considered to be the most likely catalytic base in MK0840 (described below) is located at the very edge of the first bending region, indicating interplay of domain closure and catalysis at this point in the structure. Amino acids at various positions in the three-dimensional structure have been identified to play important roles in domain closure in ASKHA kinases. For glycerol kinase, the mutagenesis of a conserved aspartic acid residue from domain I involved in MgATP binding to asparagine or alanine led to a significantly decreased enzymatic activity (Pettigrew et al., 1998). It was speculated that this residue mediates contacts between domains I and II and thus influences domain closure. This Asp corresponds to Gln44 in MK0840. Other residues lying in the CONNECT I/II region and substrate-binding region have been identified to influence domain closure in 3-phosphoglycerate kinase using SAXS, calorimetric and kinetic studies (Szabó et al., 2008a,b). These residues were found to be important for the structural integrity of the hinge region and the transmission of substrateinduced effects towards the main hinge. These and many other studies point to a cumulative effect of several side-chain interactions in the hinge region and of substrate binding in the mechanism of domain closure.

The interactions of ADP in this closed protomer $B$ (Fig. $4 c$ and Supplementary Fig. S5c) are the total of all interactions observed for both conformers of the ADP molecule in all four crystallographically independent copies of the MK0840$\mathrm{ADP}^{\text {open }}$ crystal form (Figs. $4 a$ and $4 b$ and Supplementary Figs.
$\mathrm{S} 5 a$ and $\mathrm{S} 5 b$ ). The distance between Ala168 N and Thr48 $\mathrm{O}^{\gamma 1}$ in the closed form of MK0840-ADP ${ }^{\text {closed }}$ is $4.40 \AA$, similar to the distance found in the ADP-bound form of PaAnmK (PDB entry 3qbw; $4.21 \AA$ ), and is considerably shorter than in the open form (about $8.2 \AA$ ). As a result of the domain closure, the $\alpha$-phosphates and $\beta$-phosphates are fixed in space between the two domains I and II by interactions of the phosphate $\mathrm{O}$ atoms with Ala168 $\mathrm{N}$ and Thr48 $\mathrm{O}^{\gamma 1}$ (Fig. $4 c$ and Supplementary Fig. S5c). Thus, one of the two alternating conformations of the nucleotide becomes selected upon domain closure.

\subsection{Putative substrate-binding site}

In both ADP-bound structures unaccounted-for electron density could be observed proximal to the ADP-binding site. In the $\mathrm{ADP}^{\text {open }}$ structure this density was interpreted as $\beta$-Dglucose (BGC; Figs. $4 a$ and $4 b$ and Supplementary Figs. S5a and $\mathrm{S} 5 b$ ). Owing to the lower resolution and the probable multiple binding modes, this is a tentative assignment. The glucose could originate from the sucrose solution used as a cryoprotectant. In the ADP ${ }^{\text {closed }}$ crystal form glycerol (GOL; Fig. $4 c$ and Supplementary Fig. S5c) was unequivocally identified as the ligand, originating from the crystallization solution. The ASHKA family comprises glucose and glycerol kinases and these molecules bind in the neighbourhood of the putative substrate-binding site in MK0840 as located in homologous enzymes.

The binding of BGC is essentially accomplished by watermediated hydrogen-bond interactions of the side chain of Asp146 and the backbone amide $\mathrm{N}$ atom of Asp144 with the hydroxyl group of the sugar. Additionally, Met169, Val171 and Val247 form a hydrophobic pocket around the sugar. In contrast to the water-mediated interactions with BGC in MK0840-ADP ${ }^{\text {open }}$, Asp146 and Asp144 interact directly with GOL in MK0840-ADP ${ }^{\text {closed }}$. The carboxylate O atoms of the Asp146 side chain form polar contacts to GOL O1 and O2, and the carboxylate oxygen $\mathrm{O}^{\delta 1}$ and the backbone amide $\mathrm{N}$ atom of Asp144 form contacts to GOL O2 and O3, respectively (Fig. $4 c$ and Supplementary Fig. S5c). Asp146 occupies the same spatial position as Asp237 in RhuK (Fig. 2c; Grueninger \& Schulz, 2006) and Asp211 in yeast hexokinase PII (Kuser et al., 2000), where they serve as the general base in the phosphorylation reaction. Met169 superimposes well with Trp260 of RhuK (Fig. 2c), which is rotated to fill the empty acceptor-binding site of the apoenzyme and is considered to perform a local induced fit in addition to the general domain closure during substrate binding (Grueninger \& Schulz, 2006). A similar function for Met169 can only be surmised, as the substrate preference of MK0840 is as yet unknown and only minor conformational changes are observed for the Met169 side chain in the apo and holo form.

For the following reasons, we believe that neither glucose nor glycerol bind in a substrate-like manner here: sugar kinases usually bind their substrates via several side-chainmediated or water-mediated hydrogen bonds. Here, only one such interaction is formed to BGC. In addition, BGC binding 
does not occur in one defined way (Figs. $4 a$ and $4 b$ and Supplementary Figs. S5a and S5b) in the four distinct protomers. Furthermore, the enzyme still exhibits an open conformation, whereas binding of substrates usually leads to domain closure. The same holds true for GOL, where despite the involvement of several side-chain and backbone interactions in ligand binding an open conformation is observed in one of the protomers (Fig. $4 c$ and Supplementary Fig. S5c). Such binding modes have been observed before: for glycerol kinase from P. falciparum in the ADP-bound structure (PDB entry $2 \mathrm{w} 41$ ) the cryoprotectant ethylene glycol was found in the substrate-binding site involving several interactions also found in glycerol binding (Simanshu et al., 2005; Schnick et al., 2009) and the enzyme showed an open conformation. Additionally, the binding of ASKHA substrates involves interactions from both domains I and II, as found in the complexes of RhuK with $\beta$-L-rhamnulose (PDB entry 2uyt; Grueninger \& Schulz, 2007) and $\beta$-L-fructose (PDB entry 2cgj; Grueninger \& Schulz, 2006) and the complex of $P a A n m K$ with anhMurNAc (PDB entry 3qbx; Bacik et al., 2011). Both BGC and GOL ligands only interact with residues located in domain I, leaving a larger gap between them and domain II, with a distance in the range 4.6-6.5 $\AA$ between the nearest atom of the ligand and Met169 $\mathrm{S}^{\delta}$ in domain II. To corroborate this conclusion, we performed activity assays for 45 putative substrates including GOL and BGC and none were phosphorylated (see below and Supplementary Table S1).

MK0840 is assigned as a member of the anhydro- $N$-acetylmuramic acid kinase family (Pfam PF03702; Finn et al., 2010). As mentioned above, crystal structures of this family are known for SoAnmK and $P a A n m K$. The alignment of 291 residues using DALI (Holm \& Rosenström, 2010) resulted in r.m.s.d.s of 3.9 and $3.8 \AA$ for SoAnmK and PaAnmK, respectively, for the open confirmation. However, superimposition of MK0840 $0^{\text {closed }}$ with both AnmK homologues (r.m.s.d. of $3.5 \AA$ ) shows that the architectures of their substrate-binding sites are quite different (Supplementary Fig. S2). In $P a A n m K$ the general base is reported to be Asp182 (Bacik et al., 2011), which also superposes well with Asp188 of SoAnmK. These residues occupy the same spatial position as Val187 in MK0840 (Supplementary Fig. S2) and are located on the opposite site of the putative substrate-binding site. Other residues involved in the binding of anhMurNAc, such as Arg129 in PaAnmK and the equivalent Arg134 in SoAnmK, are replaced by Asp144 in MK0840, a residue involved in glycerol binding in MK0840-ADP ${ }^{\text {closed }}$. A global comparison of the MK0840 substrate-binding pocket with ASKHA family kinase structures with highest structural similarity ( $Z$-score $>16$, r.m.s.d. $<4.4 \AA$ using $D A L I$ ) showed that one feature of the MK0840 ligand-binding pocket is quite unique. Immediately upstream of Asp144/146 there is an insertion between strand $\beta 5$ and helices $\eta 1$ and $\alpha 3$ (Fig. 2a) that harbours Trp140 and the two potassium-binding sites. It is tempting to speculate that it may have an influence on substrate binding or domain closure or even mediate allosteric regulation through Trp140, which interacts with the second protomer of the dimer.
To investigate the substrate preference of MK0840 more closely, we performed kinase assays with 45 different putative substrates (see Supplementary Material and Supplementary Table S1). For some kinases, such as pyruvate kinase M2, two different oligomeric states have been reported, of which only one represents the active form (Eigenbrodt et al., 1992). For this reason, we tested dimeric and tetrameric fractions of MK0840 for activity. None of the compounds was phosphorylated by MK0840. ATPase assays were performed in order to determine whether purified full-length and truncated MK0840 is still in an active conformation or whether it is inactivated by the purification procedure (Supplementary Material). It has been described, for example for E. coli glycerol kinase (Pettigrew et al., 1990) and yeast hexokinase (Kaji \& Colowick, 1965; DelaFuente et al., 1970), that very low substrate-independent MgATPase activity can occur in the absence of substrate. In the presence of MK0840 we observed a fivefold increase in ATP hydrolysis compared with the background (Supplementary Fig. S3). From all these findings we can conclude that MK0840 is purified in its active form but that its substrate preference remains to be elucidated.

\section{Conclusions}

The crystal structures of the putative Hsp70 metalloproteasefold MK0840 from M. kandleri reveal an ASHKA family protein that most closely resembles anhydro- $N$-acetylmuramic acid kinase. Like the ASHKA family proteins, it exhibits a bilobal architecture in which a pronounced domain closure can be observed in one crystal form, indicating a functional protein. However, structural differences are observed in the active site of MK0840, especially the lack of conservation of an aspartic acid that is crucial for catalysis in the established anhydro- $N$-acetylmuramic acid kinases. Together with the fact that M. kandleri, like other pseudomurein-coated archaea, does not contain anhMurNAc (Hartmann \& König, 1990; Kurr et al., 1991; Claus \& König, 2010), this indicates that is very unlikely that MK0840 is an anhydro- $N$-acetylmuramic acid kinase. Archaebacterial anhydro- $N$-acetylmuramic acid counterparts $(N$-acetyltalosaminuronic acid and its derivatives) are currently not available and therefore could not be tested as substrates in kinase assays. Further investigations will be necessary in order to unravel the biological function of MK0840.

This work was supported by the Swiss National Science Foundation, the University of Bern and the Berner Hochschulstiftung. The help of all of the beamline scientists at beamline X06DA at the Swiss Light Source, Paul Scherrer Institute, Villigen, Switzerland is highly appreciated.

\section{References}

Adams, P. D. et al. (2010). Acta Cryst. D66, 213-221.

Atrih, A. \& Foster, S. J. (1999). Antonie Van Leeuwenhoek, 75, 299-307.

Bacik, J.-P., Whitworth, G. E., Stubbs, K. A., Yadav, A. K., Martin, D. R., Bailey-Elkin, B. A., Vocadlo, D. J. \& Mark, B. L. (2011). J. Biol. Chem. 286, 12283-12291. 
Berman, H. M., Westbrook, J., Feng, Z., Gilliland, G., Bhat, T. N., Weissig, H., Shindyalov, I. N. \& Bourne, P. E. (2000). Nucleic Acids Res. 28, 235-242.

Bork, P., Sander, C. \& Valencia, A. (1992). Proc. Natl Acad. Sci. USA, 89, 7290-7294.

Breitung, J., Börner, G., Scholz, S., Linder, D., Stetter, K. O. \& Thauer, R. K. (1992). Eur. J. Biochem. 210, 971-981.

Brünger, A. T. (1997). Methods Enzymol. 277, 366-396.

Buss, K. A., Cooper, D. R., Ingram-Smith, C., Ferry, J. G., Sanders, D. A. \& Hasson, M. S. (2001). J. Bacteriol. 183, 680-686.

Chan, K.-M., Delfert, D. \& Junger, K. D. (1986). Anal. Biochem. 157, 375-380.

Chen, V. B., Arendall, W. B., Headd, J. J., Keedy, D. A., Immormino, R. M., Kapral, G. J., Murray, L. W., Richardson, J. S. \& Richardson, D. C. (2010). Acta Cryst. D66, 12-21.

Claus, H. \& König, H. (2010). Prokaryotic Cell Wall Compounds: Structure and Biochemistry, edited by H. König, H. Claus \& A. Varma, pp. 231-251. New York: Springer.

DelaFuente, G., Lagunas, R. \& Sols, A. (1970). Eur. J. Biochem. 16, 226-233.

DeLano, W. L. (2002). PyMOL. http://www.pymol.org.

Doublié, S. (1997). Methods Enzymol. 276, 523-530.

Eigenbrodt, E., Reinacher, M., Scheefers-Borchel, U., Scheefers, H. \& Friis, R. (1992). Crit. Rev. Oncog. 3, 91-115.

Emsley, P., Lohkamp, B., Scott, W. G. \& Cowtan, K. (2010). Acta Cryst. D66, 486-501.

Feller, G., Bussy, O., Houssier, C. \& Gerday, C. (1996). J. Biol. Chem. 271, 23836-23841.

Finn, R. D., Mistry, J., Tate, J., Coggill, P., Heger, A., Pollington, J. E., Gavin, O. L., Gunasekaran, P., Ceric, G., Forslund, K., Holm, L., Sonnhammer, E. L., Eddy, S. R. \& Bateman, A. (2010). Nucleic Acids Res. 38, D211-D222.

Frieden, C., Lieberman, D. \& Gilbert, H. R. (1980). J. Biol. Chem. 255, 8991-8993.

Gille, C. \& Frömmel, C. (2001). Bioinformatics, 17, 377-378.

Goodell, E. W. \& Schwarz, U. (1985). J. Bacteriol. 162, 391-397.

Gorrell, A., Lawrence, S. H. \& Ferry, J. G. (2005). J. Biol. Chem. 280, 10731-10742.

Gouet, P., Courcelle, E., Stuart, D. I. \& Métoz, F. (1999). Bioinformatics, 15, 305-308.

Grueninger, D. \& Schulz, G. E. (2006). J. Mol. Biol. 359, 787-797.

Grueninger, D. \& Schulz, G. E. (2007). FEBS Lett. 581, 3127-3130.

Hartmann, E. \& König, H. (1990). Naturwissenschaften, 77, 472-475.

Hayward, S. \& Berendsen, H. J. (1998). Proteins, 30, 144-154.

Holm, L. \& Rosenström, P. (2010). Nucleic Acids Res. 38, W545W549.

Hong, B. S., Yun, M. K., Zhang, Y.-M., Chohnan, S., Rock, C. O., White, S. W., Jackowski, S., Park, H.-W. \& Leonardi, R. (2006). Structure, 14, 1251-1261.

Jonge, B. L. M. de, Chang, Y.-S., Gage, D. \& Tomasz, A. (1992). J. Biol. Chem. 267, 11248-11254.

Jonge, B. L. M. de, Handwerger, S. \& Gage, D. (1996). Antimicrob. Agents Chemother. 40, 863-869.

Kabsch, W. (2010). Acta Cryst. D66, 125-132.

Kabsch, W. \& Sander, C. (1983). Biopolymers, 22, 2577-2637.

Kaji, A. \& Colowick, S. P. (1965). J. Biol. Chem. 240, 4454-4462.

Karplus, P. A. \& Diederichs, K. (2012). Science, 336, 1030-1033.

Kelley, L. A. \& Sternberg, M. J. (2009). Nature Protoc. 4, 363-371.

Krissinel, E. \& Henrick, K. (2007). J. Mol. Biol. 372, 774-797.

Kurr, M., Huber, R., König, H., Jannasch, H. W., Fricke, H., Trincone, A., Kristjansson, J. K. \& Stetter, K. O. (1991). Arch. Microbiol. 156, 239-247.

Kuser, P. R., Krauchenco, S., Antunes, O. A. \& Polikarpov, I. (2000). J. Biol. Chem. 275, 20814-20821.

Langer, G., Cohen, S. X., Lamzin, V. S. \& Perrakis, A. (2008). Nature Protoc. 3, 1171-1179.
Larkin, M. A., Blackshields, G., Brown, N. P., Chenna, R., McGettigan, P. A., McWilliam, H., Valentin, F., Wallace, I. M., Wilm, A., Lopez, R., Thompson, J. D., Gibson, T. J. \& Higgins, D. G. (2007). Bioinformatics, 23, 2947-2948.

Laskowski, R. A. \& Swindells, M. B. (2011). J. Chem. Inf. Model. 51, 2778-2786.

Locher, K. P., Hans, M., Yeh, A. P., Schmid, B., Buckel, W. \& Rees, D. C. (2001). J. Mol. Biol. 307, 297-308.

Lunin, V. V., Li, Y., Schrag, J. D., Iannuzzi, P., Cygler, M. \& Matte, A. (2004). J. Bacteriol. 186, 6915-6927.

Mamat, B., Roth, A., Grimm, C., Ermler, U., Tziatzios, C., Schubert, D., Thauer, R. K. \& Shima, S. (2002). Protein Sci. 11, 2168-2178.

McCoy, A. J., Grosse-Kunstleve, R. W., Adams, P. D., Winn, M. D., Storoni, L. C. \& Read, R. J. (2007). J. Appl. Cryst. 40, 658-674.

Morris, R. J., Perrakis, A. \& Lamzin, V. S. (2002). Acta Cryst. D58, 968-975.

Mukai, T., Kawai, S., Mori, S., Mikami, B. \& Murata, K. (2004). J. Biol. Chem. 279, 50591-50600.

Nishimasu, H., Fushinobu, S., Shoun, H. \& Wakagi, T. (2007). J. Biol. Chem. 282, 9923-9931.

Oken, M. M., Peterson, P. K. \& Wilkinson, B. J. (1981). Infect. Immun. 31, 208-213.

Painter, J. \& Merritt, E. A. (2006a). Acta Cryst. D62, 439-450.

Painter, J. \& Merritt, E. A. (2006b). J. Appl. Cryst. 39, 109-111.

Pal, D. \& Chakrabarti, P. (1999). J. Mol. Biol. 294, 271-288.

Pettigrew, D. W., Smith, G. B., Thomas, K. P. \& Dodds, D. C. (1998). Arch. Biochem. Biophys. 349, 236-245.

Pettigrew, D. W., Yu, G. J. \& Liu, Y. (1990). Biochemistry, 29, 86208627.

Qi, G. \& Hayward, S. (2009). BMC Struct. Biol. 9, 13.

Qian, M., Haser, R., Buisson, G., Duée, E. \& Payan, F. (1994). Biochemistry, 33, 6284-6294.

Scheffers, D. J. \& Pinho, M. G. (2005). Microbiol. Mol. Biol. Rev. 69, 585-607.

Schnick, C., Polley, S. D., Fivelman, Q. L., Ranford-Cartwright, L. C., Wilkinson, S. R., Brannigan, J. A., Wilkinson, A. J. \& Baker, D. A. (2009). Mol. Microbiol. 71, 533-545.

Shima, S., Hérault, D. A., Berkessel, A. \& Thauer, R. K. (1998). Arch. Microbiol. 170, 469-472.

Simanshu, D. K., Savithri, H. S. \& Murthy, M. R. (2005). J. Mol. Biol. 352, 876-892.

Slabinski, L., Jaroszewski, L., Rychlewski, L., Wilson, I. A., Lesley, S. A. \& Godzik, A. (2007). Bioinformatics, 23, 3403-3405.

Stewart-Tull, D. E. (1980). Annu. Rev. Microbiol. 34, 311-340.

Szabó, J., Varga, A., Flachner, B., Konarev, P. V., Svergun, D. I., Závodszky, P. \& Vas, M. (2008a). FEBS Lett. 582, 1335-1340.

Szabó, J., Varga, A., Flachner, B., Konarev, P. V., Svergun, D. I., Závodszky, P. \& Vas, M. (2008b). Biochemistry, 47, 6735-6744.

Terwilliger, T. C., Adams, P. D., Moriarty, N. W. \& Cohn, J. D. (2007). Acta Cryst. D63, 101-107.

Terwilliger, T. C., Grosse-Kunstleve, R. W., Afonine, P. V., Moriarty, N. W., Zwart, P. H., Hung, L.-W., Read, R. J. \& Adams, P. D. (2008). Acta Cryst. D64, 61-69.

Terwilliger, T. C., Klei, H., Adams, P. D., Moriarty, N. W. \& Cohn, J. D. (2006). Acta Cryst. D62, 915-922.

Uehara, T., Suefuji, K., Valbuena, N., Meehan, B., Donegan, M. \& Park, J. T. (2005). J. Bacteriol. 187, 3643-3649.

Van Duyne, G. D., Standaert, R. F., Karplus, P. A., Schreiber, S. L. \& Clardy, J. (1993). J. Mol. Biol. 229, 105-124.

Vriend, G. (1990). J. Mol. Graph. 8, 52-56.

Wilbanks, S. M. \& McKay, D. B. (1995). J. Biol. Chem. 270, 2251-2257.

Winn, M. D. et al. (2011). Acta Cryst. D67, 235-242.

Yang, Z. R., Thomson, R., McNeil, P. \& Esnouf, R. M. (2005). Bioinformatics, 21, 3369-3376.

Yeh, J. I., Charrier, V., Paulo, J., Hou, L., Darbon, E., Claiborne, A., Hol, W. G. J. \& Deutscher, J. (2003). Biochemistry, 43, 362-373. 